VOL. 48 (1993) [441-449]

\title{
MULTIPLIERS FOR WEIGHTED HARDY SPACES ON LOCALLY COMPACT VILENKIN GROUPS
}

\author{
TOSHIYUKI KITADA
}

Let $G$ be a locally compact Vilenkin group. We study multipliers which satisfy a generalised Hörmander condition from power-weighted Hardy space $H_{\beta}^{p}(G)$ to $H_{\beta^{\prime}}^{q}(G)$ with $0<p \leqslant q<\infty, 0<p \leqslant 1,-1<\beta, \beta^{\prime}$.

\section{Introduction and Preliminary results}

In [5] Kurtz gave weighted norm inequalities for kernel operators which map an $L^{p}\left(\mathbf{R}^{n}\right)$ space into an $L^{q}\left(\mathbf{R}^{n}\right)$ space with $1<p<q<\infty$. Applying them to multiplier operators which satisfy a generalised Hörmander multiplier condition, he obtained a multiplier theorem between weighted $L^{p}\left(\mathbf{R}^{n}\right)$ spaces and weighted $L^{q}\left(\mathbf{R}^{n}\right)$ spaces. In [11] Vinogradova considered a multiplier condition which is stronger than that of Kurtz, and gave a multiplier theorem from weighted $L^{p}\left(\mathbf{R}^{n}\right)$ space to weighted $L^{p}\left(\mathbf{R}^{n}\right)$ space with different power-weights.

In this note we consider the case $0<p \leqslant 1, p \leqslant q<\infty$ under the setting of the locally compact Vilenkin groups $G$, instead of $\mathbf{R}^{n}$. Let $H_{\beta}^{p}(0<p<\infty, \beta>-1)$ be a power-weighted Hardy space on $G$. We give a sufficient condition for a function $\varphi$ on $\Gamma$ (the dual group of $G$ ) to be a multiplier from $H_{\beta}^{p}$ to $H_{\beta^{\prime}}^{q}, 0<p \leqslant 1, p \leqslant q<\infty$. Our main result is Theorem 2, which is showed by combining multiplier theorems on $H_{\beta}^{p}(0<p<\infty, \beta>-1)$ of the present author $[2,4$ and 3$]$ with a weighted norm inequality for the fractional integral operator on $G$ (Theorem 1 ).

Throughout this note $G$ will denote a locally compact Vilenkin group, that is to say, $G$ is a locally compact abelian topological group containing a strictly decreasing sequence of compact open subgroups $\left(G_{n}\right)_{-\infty}^{\infty}$ such that

$$
\begin{aligned}
& \text { (i) } \bigcup_{-\infty}^{\infty} G_{n}=G \text { and } \bigcap_{-\infty}^{\infty} G_{n}=\{0\} . \\
& \text { (ii) } \sup \left\{\operatorname{order}\left(G_{n} / G_{n+1}\right): n \in \mathbf{Z}\right\}:=B<\infty .
\end{aligned}
$$

Received 17th December, 1992

This worl was done while the author was visiting the University of New Mexico. He would like to thank the Department of Mathematics and Statistics for its hospitality and Professor Onneweer for helpful conversations.

Copyright Clearance Centre, Inc. Serial-fee code: 0004-9729/93 $\$ A 2.00+0.00$. 
Examples of such groups are described in [1, Section 4.1.2]. Additional examples are the additive group of a local field (see [10]).

Let $\Gamma$ be the dual group of $G$ and let $\Gamma_{n}$ be the annihilator of $G_{n}$ for each $n \in Z$. Then $\left(\Gamma_{n}\right)_{-\infty}^{\infty}$ is a strictly increasing sequence of compact open subgroups of $\Gamma$ such that (i) $\bigcup_{-\infty}^{\infty} \Gamma_{n}=\Gamma$ and $\bigcap_{-\infty}^{\infty} \Gamma_{n}=\{1\}$, and (ii) order $\left(\Gamma_{n+1} / \Gamma_{n}\right)=\operatorname{order}\left(G_{n} / G_{n+1}\right)$. We choose Haar measures $d x$ on $G$ and $d \gamma$ on $\Gamma$ so that $\left|G_{0}\right|=\left|\Gamma_{0}\right|=1$, where $|A|$ denotes the Haar measure of a measurable subset $A$ of $G$, or $\Gamma$. Then $\left|G_{n}\right|^{-1}=\left|\Gamma_{n}\right|:=m_{n}$ for each $n \in \mathbf{Z}$. For $x \in G$, we set $|x|=\left(m_{n}\right)^{-1}$ if $x \in G_{n} \mid G_{n+1}$ and $|x|=0$ if $x=0$. Similarly, we set $|\gamma|=m_{n+1}$ if $\gamma \in \Gamma_{n+1} \backslash \Gamma_{n}$ and $|\gamma|=0$ if $\gamma=1$. Since $2 m_{n} \leqslant m_{n+1}$ for each $n \in \mathbf{Z}$, it follows that $\sum_{n=k}^{\infty}\left(m_{n}\right)^{-\alpha} \leqslant C\left(m_{k}\right)^{-\alpha}$ and $\sum_{n=-\infty}^{k}\left(m_{n}\right)^{\alpha} \leqslant C\left(m_{k}\right)^{\alpha}$ for any $\alpha>0, k \in \mathbf{Z}$.

The symbols $\wedge$ and $\vee$ will denote the Fourier transform and inverse Fourier transform, respectively. We have $\left(\xi_{G_{n}}\right)^{\wedge}=\left|\Gamma_{n}\right|^{-1} \xi_{\Gamma_{n}}:=F_{n}$ and, hence, $\left(\xi_{\Gamma_{n}}\right)^{\vee}=$ $\left|G_{n}\right|^{-1} \xi_{G_{n}}:=\Delta_{n}$ for each $n \in Z$, where $\xi_{A}$ denote the indicator function of a set $A$.

The Lebesgue space on $G$ with respect to the weight measure $|x|^{\alpha} d x$ will be denoted by $L_{\alpha}^{p}(G)$ or $L_{\alpha}^{p}, 0<p<\infty, \alpha \in \mathbf{R}$, and we set $\|f\|_{p, \alpha}=$ $\left(\int_{G}|f(x)|^{p}|x|^{\alpha} d x\right)^{1 / p}$. When $\alpha=0$, we write $L^{p}$ and $\|f\|_{p}$ instead of $L_{0}^{p}$ and $\|f\|_{p, 0}$, respectively. We set $|A|_{\alpha}=\int_{A}|x|^{\alpha} d x$ (hence, $|A|_{0}=|A|$ ).

Following Taibleson's development of a distribution theory on local fields [10], we define $\mathcal{S}(G)$ or $\mathcal{S}$ to be the set of all functions $\varphi$ on $G$ such that $\varphi$ has compact support and is constant on the cosets of some $G_{n}, n \in \mathbf{Z}$. A sequence $\left(\varphi_{n}\right)_{1}^{\infty}$ in $\mathcal{S}(G)$ converges to $\varphi$ in $\mathcal{S}(G)$ if there are integers $r, s$ so that each $\varphi_{n}$ and $\varphi$ are constant on the cosets of $G$, and are supported on $G_{r}$ and $\left(\varphi_{n}\right)_{1}^{\infty}$ tends to $\varphi$ uniformly on $G$. The set of all continuous linear functionals on $\mathcal{S}(G)$ will be denoted by $\mathcal{S}^{\prime}(G)$ or $\mathcal{S}^{\prime}$. A sequence $\left(f_{n}\right)_{1}^{\infty}$ in $\mathcal{S}^{\prime}(G)$ converges to $f$ in $\mathcal{S}^{\prime}(G)$ if for all $\varphi \in \mathcal{S}(G)$ we have $\lim _{n \rightarrow \infty}\left\langle f_{n}, \varphi\right\rangle=\langle f, \varphi\rangle$.

Similarly, $\mathcal{S}(\Gamma)$ and $\mathcal{S}^{\prime}(\Gamma)$ are defined. For more details, see [10].

For $f \in S^{\prime}$ we define its maximal function $f^{*}$ by $f^{*}(x)=\sup _{n}\left|f * \Delta_{n}(x)\right|$. The power-weighted Hardy spaces $H_{\alpha}^{p}:=H_{\alpha}^{p}(G)$ are defined as the space of all $f \in \mathcal{S}^{\prime}$ for which $\|f\|_{H_{\alpha}^{p}}:=\left\|f^{*}\right\|_{p, \alpha}<\infty$, where $0<p<\infty, \alpha \in \mathbf{R}$.

Let $0<p<\infty$ and $\alpha>-1$. A function $a$ on $G$ is called a $(p, \infty)_{\alpha}$ atom if there exists an interval(coset) $I:=x_{0}+G_{n}$ such that (i) supp $a \subset I$, (ii) $\|a\|_{\infty} \leqslant|I|_{\alpha}^{-1 / p}$, and (iii) $\int_{G} a(x) d x=0$. The atomic characterisation of $H_{\alpha}^{p}$ spaces are given as follows, see [6, Theorem 3.5], [3, Theorem 3.2].

LemMA 1. Let $0<p \leqslant 1$ and $-1<\alpha \leqslant 0$. Then $f \in H_{\alpha}^{p}$ if and only if 
$f=\sum_{i=1}^{\infty} \lambda_{i} a_{i}$ in $\mathcal{S}^{\prime}$, where each $\lambda_{i}>0, a_{i}$ is a $(p, \infty)_{\alpha}$ atom and $\sum_{i=1}^{\infty} \lambda_{i}^{p}<\infty$. Furthermore, $\|f\|_{H_{\alpha}^{p}} \sim \inf \left\{\left(\sum \lambda_{i}^{p}\right)^{1 / p} ; f=\sum \lambda_{i} a_{i}\right\}$.

Lemma 2. Let $1<p<\infty$ and $-1<\alpha<p-1$. Then $H_{\alpha}^{p} \cong L_{\alpha}^{p}$.

Proof: One direction of $L_{\alpha}^{p} \subset H_{\alpha}^{p}$ follows from Hardy-Littlewood maximal inequality. The other direction is seen by a routine argument. We omit the details.

Lemma 3. Let $0<p \leqslant 1$ and $-1<\alpha \leqslant 0$. Then $\mathcal{S}_{0}:=\left\{f \in \mathcal{S}, \int_{G} f(x) d x=0\right\}$ is dense in $H_{\alpha}^{p}$.

Proof: Let $f \in B_{\alpha}^{p}$ and $\varepsilon>0$. Then, by Lemma 1, there is a function $g$, which is a finite linear combination of $(p, \infty)_{\alpha}$ atoms, such that $\|f-g\|_{H_{\alpha}^{p}}^{p}<\varepsilon$. Since supp $g$ is compact and $\int g(x) d x=0$, it is easily seen that $g * \Delta_{n} \in \mathcal{S}_{0}$ for all $n \in \mathbf{Z}$. So if we show that $\left(g-g * \Delta_{n}\right)^{*} \rightarrow 0$ in $L_{\alpha}^{p}$ as $n \rightarrow \infty$, we have $\left\|f-g * \Delta_{n}\right\|_{H_{\alpha}^{p}}^{p} \leqslant$ $\|f-g\|_{H_{\alpha}^{p}}^{p}+\left\|g-g * \Delta_{n}\right\|_{H_{a}^{p}}^{p}<2 \varepsilon$ for large enough $n$, and this completes the proof of the lemma.

Since $g \in L^{1}, g * \Delta_{n}(x) \rightarrow g(x)$, for almost all $x$ as $n \rightarrow \infty$. Therefore we have

$$
\begin{aligned}
\left(g-g * \Delta_{n}\right)^{*}(x) & =\sup _{m \in \mathbf{Z}}\left|\left(g-g * \Delta_{n}\right) * \Delta_{m}(x)\right| \\
& =\sup _{m>n}\left|g * \Delta_{m}(x)-g * \Delta_{n}(x)\right| \rightarrow 0(n \rightarrow \infty),
\end{aligned}
$$

for almost all $x$. Since $\left(g-g * \Delta_{n}\right)^{*} \leqslant 2 g^{*}$ and $g * \in L_{\alpha}^{p}$, the Lebesgue dominated convergence theorem implies that $\left(g-g * \Delta_{n}\right)^{*} \rightarrow 0$ in $L_{\alpha}^{p}$.

LEMma 4. Let $\alpha>0,0<p, q<\infty$ and $\beta, \beta^{\prime}>-1$. Then there is a constant $C>0$ such that

$$
|I|^{\alpha}|I|_{\beta^{\prime}}^{1 / q} \leqslant C|I|_{\beta}^{1 / p} \text { for any interval } I,
$$

if and only if

$$
\frac{\beta}{p}-\frac{\beta^{\prime}}{q}=-\frac{1}{p}+\frac{1}{q}+\alpha \geqslant 0
$$

Proof: For $\beta>-1$, it is easy to see that $|I|_{\beta} \sim\left(m_{n}\right)^{-\beta-1}$ if $I=G_{n}, n \in \mathbf{Z}$ and $|I|_{\beta}=\left(m_{\ell}\right)^{-\beta}\left(m_{n}\right)^{-1}$ if $I=x+G_{n}, x \in G_{\ell} \backslash G_{\ell+1}, \ell<n$. The proof of the lemma follows from this fact at once.

\section{Fractional integrals and MUltipliers}

The fractional integral operator $I_{\alpha}$ on $G$ is defined by $\left(I_{\alpha} f\right)^{\wedge}(\gamma)=|\gamma|^{-\alpha} \hat{f}(\gamma), f \in$ $\mathcal{S}_{0}, \alpha>0(\operatorname{see}[10,7])$. We set $k_{\alpha}(x)=|x|^{\alpha-1}$ for $\alpha \neq 1$, and $k_{1}(x)=\log |x|$. Then, unlike the case $R^{n}, \widehat{k_{\alpha}}(\gamma)$ is not a constant times $|\gamma|^{-\alpha}$ in general. 
LEMMA 5. Let $\alpha>0$. Then, in the sense of distributions, $\widehat{k_{\alpha}}$ is a radial function on $\Gamma$ and $\widehat{k_{\alpha}}(\gamma) \sim|\gamma|^{-\alpha}$, that is, there exist constants $C_{1}, C_{2}>0$ such that

$$
C_{2}|\gamma|^{-\alpha} \leqslant\left|\widehat{k_{\alpha}}(\gamma)\right| \leqslant C_{1}|\gamma|^{-\alpha} \text { for } \gamma \in \Gamma \text {. }
$$

Proof: Consider first $\alpha \neq 1$. Since $|x|^{\alpha-1}$ is locally integrable, we have, for each $\psi \in \mathcal{S}(\Gamma)$,

$$
\begin{aligned}
\left\langle\widehat{k_{\alpha}}, \psi\right\rangle & =\left\langle|x|^{\alpha-1}, \psi^{\vee}\right\rangle \\
& =\sum_{n=0}^{\infty}\left(m_{n}\right)^{1-\alpha} \int_{G} \xi_{G_{n} \backslash G_{n+1}}(x) \psi^{\vee}(x) d x \\
& =\sum_{n=0}^{\infty}\left(m_{n}\right)^{1-\alpha} \int_{\Gamma}\left(F_{n}-F_{n+1}\right)(\gamma) \psi(\gamma) d \gamma,
\end{aligned}
$$

where $s \in \mathbf{Z}$ is an integer such that $\psi$ is constant on each cosets of $\Gamma_{s}$, but not on a coset of $\Gamma_{-+1}$ in $\Gamma$. We set $F=\sum_{n=-\infty}^{\infty}\left(m_{n}\right)^{1-\alpha}\left(F_{n}-F_{n+1}\right)$ and define

$$
\langle F, \psi\rangle:=\sum_{n=0}^{\infty}\left(m_{n}\right)^{1-\alpha} \int_{\Gamma}\left(F_{n}-F_{n+1}\right)(\gamma) \psi(\gamma) d \gamma
$$

Then $\widehat{k_{\alpha}}=F$ in $\mathcal{S}^{\prime}$. And if $\gamma \in \Gamma_{\ell+1} \backslash \Gamma_{\ell}, \ell \in \mathbf{Z}$, then

$$
\begin{aligned}
F(\gamma) & =\sum_{n=\ell+1}^{\infty}\left(m_{n}\right)^{1-\alpha}\left(F_{n}-F_{n+1}\right)(\gamma)-\left(m_{\ell}\right)^{1-\alpha} F_{\ell+1}(\gamma) \\
& =\sum_{n=\ell}^{\infty}\left(\left(m_{n+1}\right)^{1-\alpha}-\left(m_{n}\right)^{1-\alpha}\right) F_{n+1}(\gamma) \\
& =\sum_{n=\ell}^{\infty} \frac{\left(m_{n+1}\right)^{1-\alpha}-\left(m_{n}\right)^{1-\alpha}}{m_{n+1}} \\
& =|\gamma|^{-\alpha} \sum_{n=\ell}^{\infty}\left(m_{\ell+1}\right)^{\alpha} \frac{\left(m_{n+1}\right)^{1-\alpha}-\left(m_{n}\right)^{1-\alpha}}{m_{n+1}} \\
& =|\gamma|^{-\alpha} C_{\ell, \alpha}, \text { say },
\end{aligned}
$$

where the second equality follows from the fact that $\left(m_{n}\right)^{-\alpha} \rightarrow 0(n \rightarrow \infty)$. It is easy to see that

and

$$
\begin{aligned}
& \frac{1-2^{\alpha-1}}{1-B^{-\alpha}} \leqslant C_{\ell, \alpha} \leqslant \frac{1-B^{\alpha-1}}{1-2^{-\alpha}}, \quad \text { if } \alpha<1 \\
& \frac{1-B^{\alpha-1}}{1-2^{-\alpha}} \leqslant C_{\ell, \alpha} \leqslant \frac{1-2^{\alpha-1}}{1-B^{-\alpha}}, \quad \text { if } \alpha>1 .
\end{aligned}
$$

When $\alpha=1$, a similar argument for $k_{1}(x)=\log |x|$ holds and we have the conclusion of lemma. 
REMARK 1. When $m_{n}=p^{n}, n \in \mathrm{Z}(p \geqslant 2$ is a prime integer $)$, we have $\widehat{k_{\alpha}}(\gamma)=$ $\left(\left(1-p^{\alpha-1}\right) /\left(1-p^{-\alpha}\right)\right)|\gamma|^{-\alpha}, \alpha \neq 1$ and $\widehat{k_{1}}(\gamma)=\left((\log p) /\left(1-p^{-1}\right)\right)|\gamma|^{-1}$.

We consider the (generalised fractional integral) operator $T_{\alpha}$ as follows:

DEFinition 1: Let $\alpha>0$ and $\tau(\gamma)$ be a radial function on $\Gamma$ such that $\tau(\gamma) \sim$ $|\gamma|^{-\alpha}$. We define the operator $T_{\alpha}$ by $\left(T_{\alpha} f\right)^{\wedge}(\gamma)=\tau(\gamma) \widehat{f}(\gamma), f \in \mathcal{S}_{0}$. We set $\tau_{n}:=$ $\tau(\gamma), \gamma \in \Gamma_{n+1} \backslash \Gamma_{n}$, for each $n \in \mathbf{Z}$.

Note that if $\alpha>0$ and $f \in \mathcal{S}_{0}$ then $T_{\alpha} f \in \mathcal{S}_{0}$. If $0<\alpha<1$ and $f \in \mathcal{S}$ then $T_{\alpha} f$ is well defined and locally integrable. For either case, we have

$$
T_{\alpha} f=\sum_{n=-\infty}^{\infty} \tau_{n}\left(\Delta_{n+1}-\Delta_{n}\right) * f
$$

In what follows, we assume that $0<p \leqslant 1$ and $-1<\beta \leqslant 0$.

THEOREM 1. Let $\alpha>0,0<p \leqslant q<\infty$ and $\beta^{\prime}>-1$. Then the following conditions are equivalent:

$$
\begin{gathered}
\left\|T_{\alpha} f\right\|_{H_{\beta^{\prime}}^{q}} \leqslant C\|f\|_{H_{\beta}^{p}} \quad \text { for all } f \in \mathcal{S}_{0}, \\
\frac{\beta+1}{p}=\frac{\beta^{\prime}+1}{q}+\alpha \quad \text { and } 0 \leqslant \frac{1}{p}-\frac{1}{q} \leqslant \alpha .
\end{gathered}
$$

This theorem is similar to Theorem(1.5) in [8] for the fractional integral operator on $\mathbf{R}^{\mathbf{n}}$. Since our weights are power-weights, a necessary and sufficient condition for the inequality (1) is given precisely as (2). By Lemma 3, the inequality (1) has a continuous extension to all of $\boldsymbol{H}_{\beta}^{p}$.

Proof: For simplicity of notation, we write $T$ for $T_{\alpha}$.

(1) $\Rightarrow$ (2): For any interval $I:=x_{0}+G_{n_{0}}, x_{0} \in G, n_{0} \in Z$, we define $a \in \mathcal{S}_{0}$ by

$$
a(x)=(B+1)^{-1}|I||I|_{\beta}^{-1 / p}\left(\Delta_{n_{0}+1}-\Delta_{n_{0}}\right)\left(x-x_{0}\right) .
$$

Then $a$ is $a(p, \infty)_{\beta}$ atom and $\|a\|_{H_{\beta}^{p}} \leqslant 1$. And for $x \in I$,

$$
\begin{aligned}
|T a(x)| & =\left|\sum_{n=-\infty}^{\infty} \tau_{n}\left(\Delta_{n+1}-\Delta_{n}\right) * a(x)\right| \\
& =\left|\tau_{n_{0}} a(x)\right| \geqslant C\left(m_{n_{0}}\right)^{-\alpha}|I|_{\beta}^{-1 / p} \\
& =C|I|^{\alpha}|I|_{\beta}^{-1 / p} .
\end{aligned}
$$

Since $(T a)^{*} \geqslant|T a|$ on $I$, we have

$$
\begin{aligned}
1 & \geqslant\|a\|_{H_{\beta}^{p}} \geqslant C\|T a\|_{H_{\beta^{\prime}}^{q}}=C\left\|(T a)^{*}\right\|_{q, \beta^{\prime}} \\
& \geqslant C\|T a\|_{q, \beta^{\prime}} \geqslant C|I|^{\alpha}|I|_{\beta}^{-1 / p}|I|_{\beta^{\prime}}^{1 / q} .
\end{aligned}
$$


Hence, Lemma 4 implies (2).

(2) $\Rightarrow(1)$ : We first show that for $(p, \infty)_{\alpha}$ atom $a, T a$ is a $(q, \infty)_{\beta^{\prime}}$ atom up to a constant which is independent of $a$. Let $a$ be a $(p, \infty)_{\beta}$ atom such that supp $a \subset I:=$ $x_{0}+G_{n_{0}}, x_{0} \in G, n_{0} \in \mathbf{Z}$. If $x \notin I$, then $x-x_{0} \in G_{\ell} \backslash G_{\ell+1}$ for some $\ell \in \mathbf{Z}, \ell<n_{0}$. Then $\left(x+G_{n}\right) \cap I=\emptyset$ for $n>\ell$, and $x+G_{n} \supset I$ for $n \leqslant \ell$. So $\Delta_{n} * a(x)=0$ for all $n \in \mathbf{Z}$. This shows $T a(x)=0$. Hence, supp $T a \subset I$.

Let $x \in I$. If $n<n_{0}$, then $x+G_{n+1} \supset I$ and $\Delta_{n+1} * a(x)=0$. Hence, by Lemma 4,

$$
\begin{aligned}
|T a(x)| & \leqslant \sum_{n=n_{0}}^{\infty}\left|\tau_{n}\left(\Delta_{n+1}-\Delta_{n}\right) * a(x)\right| \\
& \leqslant C \sum_{n=n_{0}}^{\infty}\left(m_{n}\right)^{-\alpha}\|a\|_{\infty} \leqslant C\left(m_{n_{0}}\right)^{-\alpha}\|a\|_{\infty} \\
& \leqslant C|I|^{\alpha}|I|_{\beta}^{-1 / p} \leqslant C|I|_{\beta^{\prime}}^{-1 / q} .
\end{aligned}
$$

The cancellation property of $T a$ follows from that of $a$. Therefore $T a$ is a $(q, \infty)_{\beta^{\prime}}$ atom up to a constant such that

$$
\|T a\|_{H_{\beta^{\prime}}^{q}} \leqslant C
$$

where $C$ is independent of $a$ (we note that under the condition (2), $\beta^{\prime} \leqslant q(1 / p-\alpha)-$ $1 \leqslant 0$, so by Lemma $2,\|T a\|_{H_{\beta^{\prime}}^{q}} \sim\|T a\|_{q, \beta^{\prime}}$, if $q>1$ ).

The inequality (3) also holds for the modified operator $T^{N}(N \in \mathbf{Z})$ defined by $\left(T^{N} f\right)^{\wedge}=\tau \xi_{\Gamma \backslash \Gamma_{N}} \hat{f}$. This is checked easily and we emphasise that the constant $C$ in (3) for $T^{N}$ is the same as the one in (3) for $T$.

Let us go on to prove (1). We consider the case $q \leqslant 1$ and $q>1$ separately. In either case, for $f \in \mathcal{S}_{0}$, let $f(x)=\sum_{i=1}^{\infty} \lambda_{i} a_{i}$ be a possible atomic decomposition of $f$ (as an element of $B_{\beta}^{p}$ ). Since $f \in \mathcal{S}_{0}$, there is an $N \in \mathbf{Z}$ such that $\hat{f}=0$ on $\Gamma_{N}$. Then we have $T f=T^{N} f$.

If $q \leqslant 1$, then it follows from (3) that

$$
\left\|\sum_{i=1}^{\infty} \lambda_{i} T^{N} a_{i}\right\|_{H_{\beta^{\prime}}^{q}} \leqslant C\left(\sum_{i=1}^{\infty} \lambda_{i}^{q}\right)^{1 / q} \leqslant C\left(\sum_{i=1}^{\infty} \lambda_{i}^{p}\right)^{1 / p}
$$

since $p \leqslant q$. This means that $\sum_{i=1}^{\infty} \lambda_{i} T^{N} a_{i}$ converges in $H_{\beta^{\prime}}^{q}$. Hence, for any $\psi \in \mathcal{S}(\Gamma)$, we have

$$
\left\langle\left(\sum_{i=1}^{\infty} \lambda_{i} T^{N} a_{i}\right)^{\wedge}, \psi\right\rangle=\left\langle\sum_{i=1}^{\infty} \lambda_{i} \widehat{a}_{i} \tau \xi_{\Gamma \backslash \Gamma_{N}}, \psi\right\rangle=\left\langle\sum_{i=1}^{\infty} \lambda_{i} \widehat{a}_{i}, \tau \xi_{\Gamma \backslash \Gamma_{N}} \psi\right\rangle
$$


because $\tau \xi_{\Gamma \backslash \Gamma_{N}}$ is locally constant on $\Gamma$,

$$
\begin{aligned}
& =\left\langle\left(\sum_{i=1}^{\infty} \lambda_{i} a_{i}\right)^{\wedge}, \tau \xi_{\Gamma \backslash \Gamma_{N}} \psi\right\rangle=\left\langle\tau \xi_{\Gamma \mid \Gamma_{N}} \widehat{f}, \psi\right\rangle \\
& =\left\langle\left(T^{N} f\right)^{\wedge}, \psi\right\rangle=\langle\widehat{T f}, \psi\rangle .
\end{aligned}
$$

Therefore we have $T f=\sum_{i=1}^{\infty} \lambda_{i} T^{N} a_{i}$ and

$$
\|T f\|_{H_{\beta^{\prime}}^{q}} \leqslant C\left(\sum_{i=1}^{\infty} \lambda_{i}^{p}\right)^{1 / p} .
$$

By taking the infimum on the right hand side above, we have the inequality (1).

If $q>1$, then by using Minkowsky's inequality,

$$
\begin{aligned}
\left(\int_{G}\left|\sum_{i=1}^{\infty} \lambda_{i} T^{N} a_{i}(x)\right|^{q}|x|^{\beta^{\prime}} d x\right)^{1 / q} & \leqslant \sum_{i=1}^{\infty} \lambda_{i}\left(\int_{G}\left|T^{N} a_{i}(x)\right|^{q}|x|^{\beta^{\prime}} d x\right)^{1 / q} \\
& \leqslant C \sum_{i=1}^{\infty} \lambda_{i} \leqslant C\left(\sum_{i=1}^{\infty} \lambda_{i}^{p}\right)^{1 / p}
\end{aligned}
$$

because $p \leqslant 1$. Hence, $\sum_{i=1}^{\infty} \lambda_{i} T^{N} a_{i}$ converges in $L_{\beta^{\prime}}^{q}$. The remainder of the proof is the same as the case $q \leqslant 1$. This completes the proof of theorem.

REMarK 2. Compared with the proof of Theorem(1.5) in [9], our proof of Theorem 1 is simple as above. It is due to the fact that $\mathcal{S}_{0}(G)=\{f \in \mathcal{S}(G)$, supp $\hat{f} \notin 1\}$. In [9] Strömberg and Wheeden also deal with the case $p>1$, and obtain [9, Theorem (1.1)]. For the groups $G$, by using other methods as in [8], we can get the following result:

Let $1<p \leqslant q<\infty, 0<\alpha<1$ and $-1<\beta<p-1,-1<\beta^{\prime}$. Then

if and only if

$$
\left\|T_{\alpha} f\right\|_{q, \beta^{\prime}} \leqslant C\|f\|_{p, \beta} \quad \text { for all } f \in \mathcal{S}
$$

$$
\frac{\beta+1}{p}=\frac{\beta^{\prime}+1}{q}+\alpha \quad \text { and } 0 \leqslant \frac{1}{p}-\frac{1}{q} \leqslant \alpha .
$$

Before stating Theorem 2, we need to introduce a generalised Hörmander class of multipliers space, $M(s, \lambda, \alpha)$ (see [5]). 
Definition 2: Let $\lambda>0,1 \leqslant s \leqslant \infty$ and $\alpha \in \mathbf{R}$. For a function $\varphi$ on $\Gamma$, we set $\varphi_{j}:=\varphi \xi_{j+1} \backslash \Gamma_{j}, j \in \mathrm{Z}$. A function $\varphi$ on $\Gamma$ belongs to $M(s, \lambda, \alpha)$ if there is a constant $C$ such that

$$
|\varphi(\gamma)| \leqslant C|\gamma|^{-\alpha} \text { and } \sup _{j \in \mathbf{Z}}\left\{\left(m_{j}\right)^{\lambda-1 / \bullet+\alpha}\left\|D^{\lambda} \varphi_{j}\right\|_{\bullet}\right\}<\infty
$$

where $D^{\lambda} \varphi_{j}:=\left(|x|^{\lambda}\left(\varphi_{j}\right)^{\vee}\right)^{\wedge}$.

$M(s, \lambda, 0)$ is $M(s, \lambda)$ introduced in [2, and 3]. Notice that if we let $\varphi(\gamma)=$ $\psi(\gamma)|\gamma|^{-\alpha}$, then $\varphi \in M(s, \lambda, \alpha)$ if and only if $\psi \in M(s, \lambda)$. Also, $|\gamma|^{-\alpha} \in M(s, \lambda, \alpha)$ for all $\lambda>0$ and $1 \leqslant s \leqslant \infty$.

TheOREM 2. Let $\alpha>0$ and $0 \leqslant 1 / p-1 / q \leqslant \alpha$. Suppose that $\varphi \in M(s, \lambda, \alpha)$ for $1 \leqslant s \leqslant \infty, \lambda>\max (1,1 / q)-1 / \max \left(2, s^{\prime}\right)$. Then

$$
\left\|(\varphi \hat{f})^{\vee}\right\|_{H_{\beta^{\prime}}^{q}} \leqslant C\|f\|_{H_{\beta}^{p}} \text { for all } f \in \mathcal{S}_{0},
$$

if $-1<\beta \leqslant 0, \max (-1,-q \lambda)<\beta^{\prime}$ and

$$
\frac{\beta+1}{p}=\frac{\beta^{\prime}+1}{q}+\alpha
$$

Proof: Let $\psi(\gamma):=\varphi(\gamma)|\gamma|^{\alpha}$ and $f \in \mathcal{S}_{0}$. Then $\psi \in M(s, \lambda)$.

If $q \leqslant 1$, then, by Theorem 4.5 in [3] and Theorem 1, we have

$$
\begin{aligned}
\left\|(\varphi \hat{f})^{\vee}\right\|_{H_{\beta^{\prime}}^{q}} & =\left\|\left(\psi\left(I_{\alpha} f\right)^{\wedge}\right)^{\vee}\right\|_{H_{\beta^{\prime}}^{q}} \\
& \leqslant C\left\|I_{\alpha} f\right\|_{H_{\beta^{\prime}}^{q}} \\
& \leqslant C\|f\|_{H_{\beta}^{p}} .
\end{aligned}
$$

If $q>1$, then, by Theorem 1 in [2] or Theorem(3.6) in [4] and Theorem 1, we have,

$$
\left\|(\varphi \hat{f})^{\vee}\right\|_{H_{\beta^{\prime}}^{q}} \leqslant C\left\|I_{\alpha} f\right\|_{H_{\beta^{\prime}}^{q}} \leqslant C\|f\|_{H_{\beta}^{p} .} .
$$

This completes the proof of theorem.

By Lemma 3, the inequality (4) in Theorem 2 has a continuous extension to all of $H_{\beta}^{p}$. When $0<\alpha<1$, we can prove Theorem 2 directly by the method as in the proof of [3, Theorem 4.4 and Theorem 4.5].

- For the case $p>1$, we can also get a similar result to Theorem 2 by the same idea as in the proof above (see Remark 2). This will appear elsewhere. 


\section{REFERENCES}

[1] R.E. Edwards and G.I. Gaudry, Littlewood-Paley and multiplier theory (Springer Verlag, Berlin, Heidelberg, New York, 1977).

[2] T. Kitada, 'Weighted $L^{p}$ multipliers on locally compact Vilenkin groups', Sci. Rep. Hirosaki Univ. 36 (1989), 135-143.

[3] T. Kitada, 'Weighted $H^{p}$ multipliers on locally compact Vilenkin groups', Monatsh. Math. 110 (1990), 283-295.

[4] T. Kitada and C.W. Onneweer, 'Hörmander-type multipliers on locally compact Vilenkin groups', in Theory and applications of Gibbs derivatives (Institute of Mathematica, Belgrade, 1989).

[5] D.S. Kurtz, 'Sharp function estimates for fractional integrals and related operators', $J$. Austral. Math. Soc. Ser. A 49 (1990), 129-137.

[6] C.W. Onneweer and T.S. Quek, 'Multipliers on weighted Hardy spaces over locally compact Vilenkin groups, I', J. Austral. Math. Soc. Ser. A 48 (1990), 472-496.

[7] L. Saloff-Coste, 'Opérateurs pseudo diifférentiels sur certains groupes totalement discontinus', Studia Math. 83 (1986), 205-228.

[8] E.T. Sawyer and R.L. Wheeden, 'Weighted inequalities for fractional integrals on Euclidian spaces and homogeneous spaces'. (Preprint 1989).

[9] J-O. Strömberg and R.L. Wheeden, 'Fractional integrals on weighted $H^{p}$ and $L^{p}$ spaces', Trans. Amer. Math. Soc. 287 (1985), 293-321.

[10] M.H. Taibleson, Fourier analysis on local fields, Mathematical Notes 15 (Princeton University Press, 1975).

[11] G.A. Vinogradova, 'On multipliers in weighted spaces', Anal. Math. 16 (1990), 215-226.

Department of Mathematics

Faculty of General Education

Hirosaki University

Hirosaki 036

Japan 